\title{
A Case Report of an Abdominal Wall Endometrioma
}

\author{
Saima Najam ${ }^{1}$, Messaoud Bounnah ${ }^{2}$ \\ ${ }^{1}$ FCPS, PG Certification in Medical Education (Dundee), Head of the OBGYN Department, \\ Dr. Sulaiman Al Habib Hospital, Sweidi, Riyadh, Saudi Arabia \\ ${ }^{2}$ Consultant Surgeon, Dr. Sulaiman Al Habib Hospital, Sweidi, Riyadh, Saudi Arabia
}

Corresponding Author: Saima Najam

\begin{abstract}
Abdominal wall endometrioma (AWE) is one of the rarest forms of the endometriosis, and usually found in the young patients, average age is 35years. The most common triad of the symptoms is, abdominal mass at or near the scar, cyclical pain and history of previous caesarean or any other gynecological surgery. Exact pathogenesis is unknown as average time of its presentation in the patients is highly variable from 3 months till 2 decades. It can be suspected on ultrasonography and CT scan and MRI are the modalities used to assess and stage the lesion prior to the surgery. Histopathological diagnosis is confirmatory. We are reporting 30 years old, Para 2, with history of first caesarean section 6 years ago followed by the normal delivery. She presented with the history of cyclical abdominal pain with the feeling of abdominal lump from last 3 months. On examination a slightly tender $5 * 4 \mathrm{~cm}$ extra abdominal mass was palpable above the right half of the previous transverse scar of the caesarean. After evaluation endometriosis was suspected and the patient opted for the surgical resection of the mass. The mass was removed in collaboration with the surgeons and the histopathology confirmed the diagnosis of the AWE.

Conclusion: AWE is a dynamic yet incompletely known entity as a result of increasing number of the caesarean and gynecological procedures. It should be kept in the differential diagnosis of the patients coming with the cyclical abdominal pain and abdominal lump with the history of previous uterine surgery. Ultrasound and MRI are the main aiding modalities but the confirmation of the diagnosis is made only after histopathology. The surgical removal of the mass with negative margins represents the current best treatment.
\end{abstract}

Key Words: Abdominal wall, endometriosis, magnetic resonance imaging, ultrasonography

\section{INTRODUCTION}

Endometriosis is characterized by the presence of the endometrial and stromal cells located at ectopic sites. It is considered as a benign inflammatory disease, which is estrogen dependent. ${ }^{1}$ It is one of the common gynecological entities with incidence of $8-18 \% .{ }^{2}$ Most common pelvic location of the endometriosis is the ovary and the pelvic peritoneum. Sites of the extra pelvic locations include gastrointestinal tract, urinary tract and the respiratory system. ${ }^{3-5}$ Among these one of the rarest forms of the endometriosis is the abdominal wall endometriosis. (AWE), which is an uncommon aftermath of gynecological surgeries such as caesarean section or an abdominal hysterectomy. ${ }^{1}$ Its incidence after caesarean section is $0.03-0.4 \%{ }^{6}$

Treatment modalities include hormonal therapies and the surgical excision. However wide surgical excision is the treatment of choice in the literature. ${ }^{7}$

\section{CASE REPORT}

We are reporting a case of 30 years old middle eastern woman, who was married for 8 years and was para two. Her 
first baby boy was delivered at term by caesarean section 6 years ago due to fetal distress manifested by the cardiotocographic abnormalities. The second baby was delivered as spontaneous vertex delivery at term after 2 years of the caesarean section. Her cycle was regular and she was not using any contraception. She presented with the history of abdominal pain off and on from last three months, the pain was dull and progressive in nature and is more intense at the end of the cycle. The pain was localized above the right margin of the previous scar. No radiation of the pain was observed, and the menstruation was the only aggravating factor noted by the patient. On examination nothing significant was observed on inspection except the previous cesarean scar. On palpation mild tenderness was noted and an extra abdominal tender mass of $5 * 4 \mathrm{~cm}$ was palpable above the right half of the previous scar. It was not attached to the overlying skin. It had restricted mobility with the suspicion of the invasion in to the muscles. Detailed counseling was done of the patient for the selection of the most appropriate investigation and the patient opted for magnetic resonance imaging (MRI). On MRI a 2.7 x 3.7 x 3.5 cm multi lobulated cystic lesion was seen embedded within the abdominal wall muscles at the level of the right side of the cesarean section scar. It had bright $\mathrm{T} 1$ and $\mathrm{T} 2$ signals. It represents endometrioma that invades the right rectus abdominis muscle. Post contrast injection showed no solid internal enhancement.

Junctional zone measured $1.2 \mathrm{~cm}$ in keeping with adenomyosis. There was no pelvic endometriosis or ovarian endometrioma. Cervix, vagina, myometrium, adnexa, ovaries, and partially distended urinary bladder were all unremarkable. There was no peritoneal or omental nodule or lymphadenopathy. Visualized skeleton, bowel loops and upper abdominal organs reveal no suspicious focal abnormality, as shown in figure 1 and 2 .

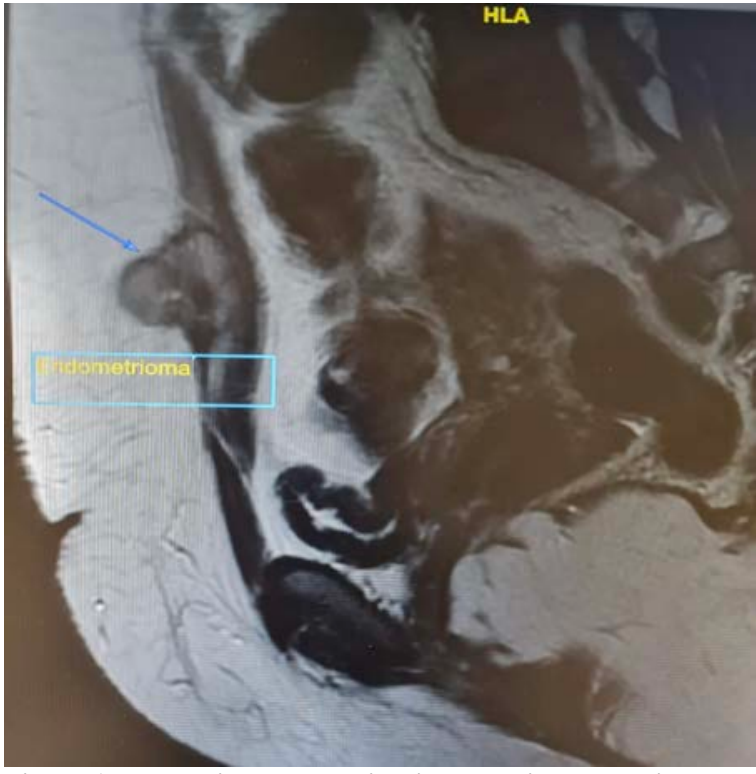

Figure-1: MRI image, longitudinal section: showing the endometrioma.



Figure-2: MRI image, transverse section: showing the endometrioma.

The treatment options discussed with the patient and the patient opted for the surgery.

In collaboration with the surgeons the surgery was done. Under general anesthesia the patient was put in dorsal position. Area was cleaned and draped and the right transverse incision was given 2.0 $\mathrm{cm}$ below the mass. A tri lobed endometriotic mass that started in the subcutaneous fat and continued deep on the aponeurosis of the right external oblique 
muscle and medially on the anterior sheath of the right rectus muscle was observed. The muscle itself was not caught by the mass. A strip of the aponeurosis was excised with the mass to ensure the disease-free margins as shown in figure3. As a result of the excision of the mass a small defect was created which was closed easily with the series of tension free interrupted absorbable sutures. Hemostasis was secured and the skin was closed. Aseptic dressing was done. Her immediate post op recovery was uneventful and the patient was discharged on first post op day in stable condition.

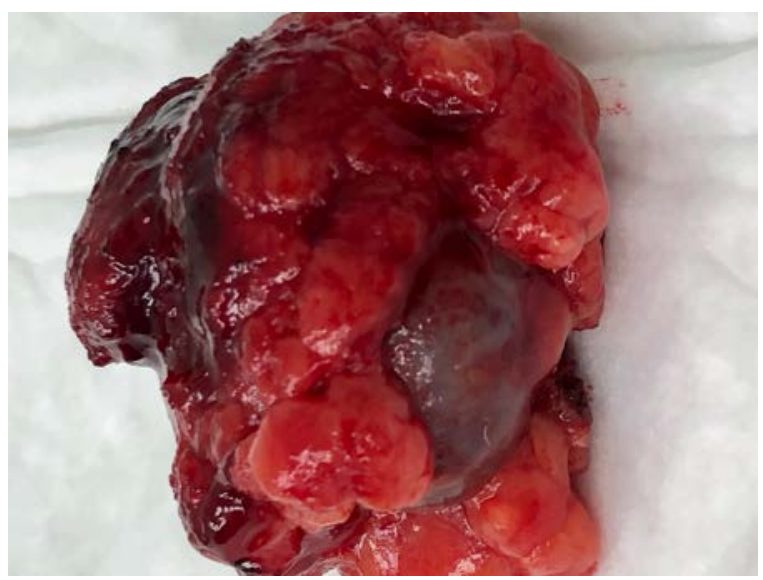

Figure 3: The gross appearance of the mass after removal.

The patient presented in the outpatient department after a week with the histopathology report, which showed endometrial glands and the stroma with clear resected margins. Hence endometriosis was confirmed.

On examination of her wound, there was no stroma at the operative site and the wound was healing well. She was followed up for two months and the complete relief of the symptoms was observed.

\section{DISCUSSION}

Endometriosis is described as the ectopic endometrial tissue outside the uterine cavity. ${ }^{8}$

The mean age of diagnosis is 35 years and the time of the surgery to development and diagnosis of endometriosis varies from 3 months till 2 decades. ${ }^{9}$ The patient in the current report developed the disease after 6 years of the previous caesarean. Although AWE is considered as iatrogenic subtype of the endometriosis, the clear explanation for the development of the disease, in some patients only is unclear. In additional to the operative technique and precautions it seems that the pathogenic mechanism is more complex.10 The clinical triage usually includes the cyclical abdominal pain and the lump at or near the scar with the history of caesarean section or similar gynecological procedures. ${ }^{4,11}$ Our patient was 30 years of age and had the same triage of the symptoms.

The ultrasonography and the Doppler can help in the pre-operative diagnosis and can show an image with fluid or mixed content depending on the phase of the menstrual cycle. ${ }^{12} \mathrm{CT}$ and MRI are helpful not only in diagnosis but also helps to detect the depth of the lesion. The sensitivity and specificity of the MRI for the diagnosis of the endometriosis is 90 and $98 \%$ respectively and in addition it has better contrast resolution then CT and ultrasound. ${ }^{13}$ The patient under discussion opted for MRI after counseling about the strengths and the limitation of each modality. Different treatment options are available, medical treatment include oral contraceptive pills with progesterone, antiestrogens such as danazol, and gonadotropic agonists like leuprolide acetate. ${ }^{14}$ but unfortunately the success rate of the medical treatment is very poor, eventually necessitating surgery in most of the patients. 15 The medical treatment can be used as an induction treatment aiming to reduce the size of the endometrioma and reduction in the parietal defect resulting from the surgical excision. ${ }^{16}$ The treatment of the choice for the AWE is wide resection of the lesion including $5-10 \mathrm{~mm}$ of the surrounding healthy tissue to ensure complete surgical resection with negative margins. Careful resection is needed to prevent rupture of the mass to avoid reimplantation of microscopic remnants of endometrial tissue. ${ }^{6}$ The parietal reconstruction after excision sometimes requires the polypropylene mesh, which was not required in the case 
understudy, and the surgeons were able to do the end-to-end closure.

\section{CONCLUSION}

AWE is a dynamic yet incompletely known entity as a result of increasing number of the caesarean and gynecological procedures. It should be kept in the differential diagnosis of the patients coming with the cyclical abdominal pain and abdominal lump with the history of previous uterine surgery. Ultrasound and MRI are the main aiding modalities but the confirmation of the diagnosis is made only after histopathology. The surgical removal of the mass with negative margins represents the current best treatment.

\section{ACKNOWLEDGEMENT}

The authors are indebted to the surgical team for the co management.

\section{Conflict of Interest: None}

\section{Source of Funding: None}

\section{REFERENCES}

1. Vagholkar K, Vagholkar S. Abdominal wall endometrioma: A diagnostic Enigma-A case report and the review of the Literature. Case Rep Obstet Gynecol, 2019;2019:6831545.

2. Kumar RR. Spontaneous abdominal wall endometriosis: A case report. Int J. Surg. Case Rep.2021;78:180-3.

3. Lainas P, Dammaro C, Rodda GA, Dagher I. Appendiceal endometriosis invading the sigmoid colon: a rare entity. Int J Colorectal Dis. 2019;34:1147-50.

4. Alsinan TA, AlDahleh LA, Alreefi HAA, Albiabi SA,Alsouss YO,Alshurafa ZH, et al. Endometriosis of the urinary bladder causing a right hydroneprosis: A case report. Am J Case Rep. 2019;14:1360-3

5. Tong SS, Yin XY, Hu SS, Cui Y, Li HT. Case report of pulmonary endometriosis and review of Literature. J Int Med Res.2019; 47:1766-70.

6. Gupta P, Gupta S. Scar endometriosis: a case report with literature review. Acta Med Iran. 2015;53(12):793-5.
7. Bektas H, Bilsel Y, Sari YS, Ersoz F, Koc $\mathrm{O}$, Deniz $\mathrm{M}$, et al. Abdominal wall endometrioma; a 10-year experience and brief review of the literature. J Surg Res. 2010;164:77-81.

8. Goel P, Sood SS, Dalal A, Romilla. Caesarean scar endometriosis- report of two cases. Indian J Med Sci.2005;59:495-8.

9. Pas K, Joanna SM, Renata R,Skret A, Barnas E. Prospective study concerning 71 cases of caesarean scar endometriosis. (CSE). J Obstet Gynaecol. 2017;37:775-8.

10. Carsote M, Terzea DC, Valea A, Galateanu AAG. Abdominal wall endometriosis ( a narrative review). Int $\mathrm{J}$ Med Sci.2020; 17(4):536-42.

11. Tatli F, Gozeneli O, Uyanikoglu H,Uzunkoy A,Yalcin HC,Ozgonul A, et al.The clinical characteristics and surgical approach of scar endometriosis: A case series of 14 women. Bosn J Basic mmed Sci.2018;18:275-8.

12. Jayi S, Laadioui M, Bouguern $\mathrm{H}$, Chaara H,Melhouf A. L'endomtriose de la paroi abdominale: a propos d'un as rare. Pan Afr Med J.2013;15:86-90.

13. Balleyguier C, Chapron C, Chpin N, Helenon O, Menu Y. Abdominal wall and surgical scar endometriosis: results of magnetic resonance imaging. Gynecol Obstet Invest.2003;55:220-4.

14. Rivlin ME, Das SK, Patel RB, Meeks GR. Leuprolide acetate in the management of cesarean scar endometriosis. Obstet and Gynecol.1995;85:838-9.

15. Khamechian T, Alizargar J, Mazoochi T. 5Year data analysis of patients following abdominal wall endometrioma surgery. BMC Womens Health.2014;14:151-5.

16. Slaiki S, Jamor J. Endometriosis of the rectus abdominis muscles: a rare case of dual location. J of Surg Case Rep.2020 September 9, cited 2021 October 26. Available from http://doi.org/10.1093/jscr/rjaa360.

How to cite this article: Najam S, Bounnah M. A case report of an abdominal wall endometrioma. Int J Health Sci Res. 2021; 11(11): 261-264. DOI: https://doi.org/10.52403 /ijhsr.20211131 